\title{
Medullary Carcinoma of the Colon: A Histopathologic Challenge
}

\author{
Zainab Fatima ${ }^{1}$, Purva Sharma ${ }^{2}$, Bahaaeldin Youssef ${ }^{3}$, Koyamangalath Krishnan ${ }^{2}$ \\ 1. Internal Medicine, East Tennessee State University, Johnson City, USA 2. Medical Oncology, East Tennessee State \\ University, Johnson City, USA 3. Pathology, East Tennessee State University, Johnson City, USA
}

Corresponding author: Zainab Fatima, zainab_fatima101@hotmail.com

\begin{abstract}
Medullary carcinoma (MC) of the colon is a rare and unique histologic subtype of colorectal cancer. It is commonly associated with deficient mismatch repair proteins and has a strong association with Lynch syndrome. Diagnosis is challenging as it does not have the usual immunohistochemical stains on pathology seen in colorectal adenocarcinoma. Here, we discuss an interesting case of MC of the colon that was metastatic on presentation and constituted a diagnostic challenge.
\end{abstract}

Review began 05/22/2021 Review ended 06/14/2021 Published 06/22/2021

๑) Copyright 2021

Fatima et al. This is an open access article distributed under the terms of the Creative Commons Attribution License CC-BY 4.0., which permits unrestricted use, distribution, and reproduction in any medium, provided the original author and source are credited.
Categories: Internal Medicine, Pathology, Oncology

Keywords: medullary carcinoma, colon cancer, chemotherapy, metastasis, microsatellite instability, immunohistochemistry

\section{Introduction}

Medullary carcinoma (MC) of the colon is a rare subtype of colon carcinoma which includes poorly differentiated and undifferentiated histology. The incidence of MC is estimated to be $0.03 \%$ of all colorectal carcinomas (CRC) [1]. MC is twice as prevalent in middle-aged women, favors the right side of the colon, and has a lower risk of lymph node metastasis than colon adenocarcinoma. MC can be poorly differentiated (72\%) or undifferentiated (22\%) with solid sheets of cells and excessive intraepithelial lymphocytic infiltration [2]. It does not have the glandular structure seen in adenocarcinoma [2]. Only a few cases of metastatic MC have been published in the literature [2,3]. We present a rare case of $\mathrm{MC}$, which was metastatic at presentation, and the diagnostic challenge it posed as the tumor cells lacked the usual markers for intestinal differentiation, such as cytokeratin (CK)20 and special AT-rich sequence binding protein 2 (SATB2). Loss of mismatch repair proteins and expression of calretinin helped validate the diagnosis.

\section{Case Presentation}

A 77-year-old female with a history of hypertension, hyperlipidemia, and osteopenia presented with a fourmonth history of progressively worsening lower abdominal pain. Abdominal pain radiated to the back and was associated with vomiting and abdominal distention. The patient also reported excessive fatigue and weight loss of 10 pounds over two months. Physical examination was significant for diffuse abdominal tenderness with rebound tenderness in the right upper quadrant and periumbilical region.

Laboratory evaluation was unremarkable, including complete blood count and chemistry panel. Computed tomography (CT) scan of the abdomen/pelvis with contrast showed a $9.1 \times 4.0 \mathrm{~cm}$ mass in the right lower quadrant, associated with thickening of the anterior wall of the cecum with mesenteric and retroperitoneal nonbulky lymphadenopathy (Figures 1,2). 


\section{Cureus}

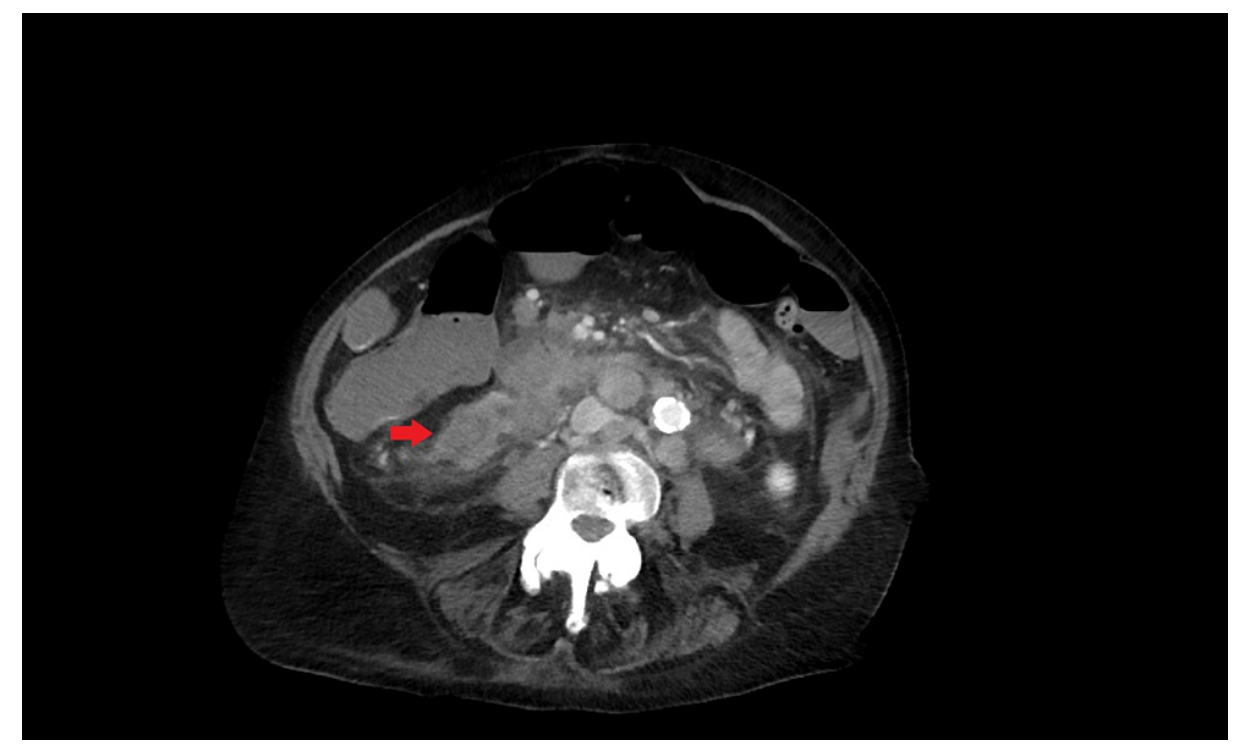

FIGURE 1: Enlarged right lower quadrant mass measuring $9.1 \times 4.0 \mathrm{~cm}$.

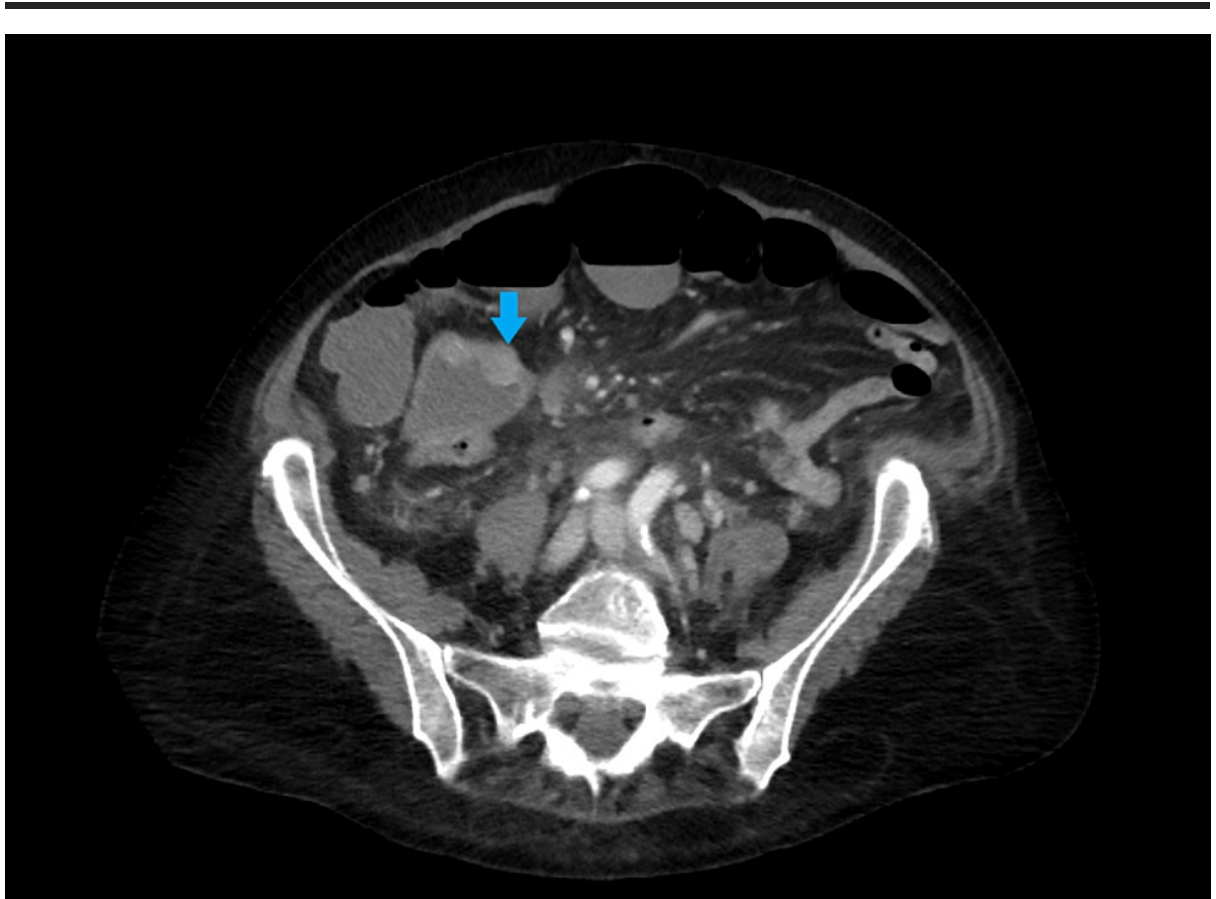

FIGURE 2: Nodular thickening in the cecum.

CT also showed an enlarged para-aortic lymph node measuring $3.1 \mathrm{~cm}$ and distal paraesophageal lymphadenopathy measuring $1.7 \mathrm{~cm}$ on the largest dimension (Figure 3). 


\section{Cureus}

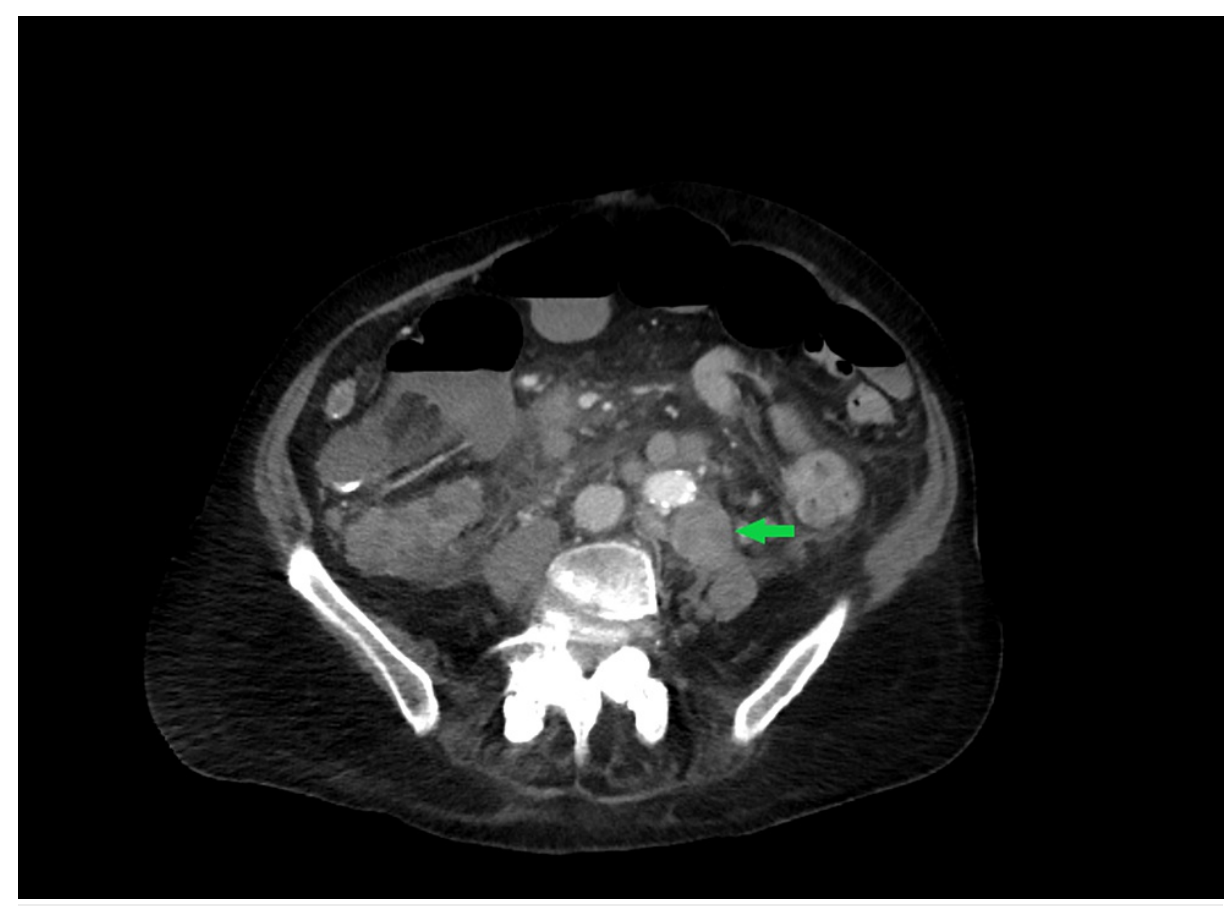

FIGURE 3: Enlarged para-aortic lymph node measuring $3.1 \mathrm{~cm}$.

Given the extensive abdominal lymphadenopathy and concern for a lymphoproliferative disorder, a bone marrow biopsy was done, which ruled out underlying bone marrow disorder. Staging workup using CT chest with contrast showed moderate bilateral pleural effusions. Colonoscopy was inconclusive due to the inability to advance the scope into the cecum because of excessive bowel looping. However, no masses were observed in the large intestine. Carcinoembryonic antigen was within normal limits $(0.9 \mathrm{ng} / \mathrm{mL})$. CT-guided biopsy of the right lower quadrant mass showed poorly differentiated malignant neoplasm with infiltrative pleomorphic cells. On immunohistochemistry (IHC), the cells were positive for CKAE1/AE3, GATA binding protein 3 (GATA3), calretinin, p63, and caudal-type homeobox 2 (CDX2) and negative for CK7, CK20, and SATB2 (Figure 4). 


\section{Cureus}

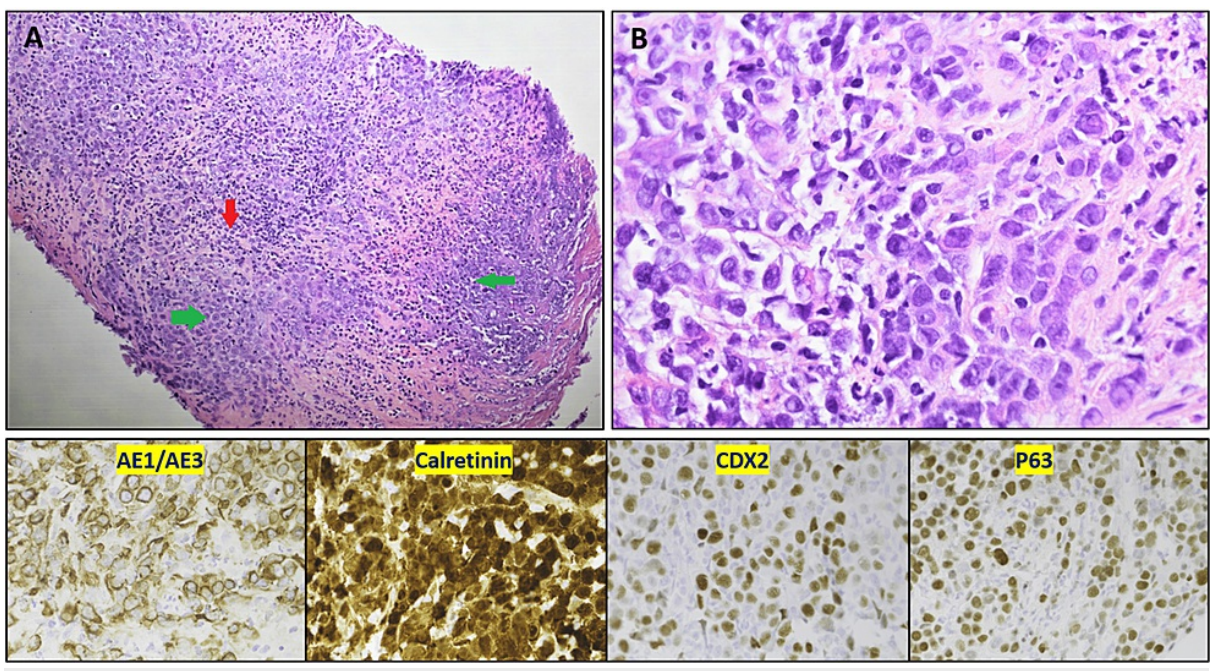

FIGURE 4: H\&E stain of the biopsy at low magnification reveals sheets of poorly differentiated cells (green arrow) in a background of necrosis with abundant intratumoral lymphocytes (red arrow) (A). Higher magnification reveals moderately to severely pleomorphic cells with medium-sized hyperchromatic nuclei, prominent nucleoli, and moderate eosinophilic cytoplasm (B). Immunohistochemically, the tumor cells were positive for CKAE1/AE3, calretinin, CDX2, and p63.

H\&E: hematoxylin and eosin; CKAE1/AE3: cytokeratin AE1/AE3; CDX2: caudal-type homeobox 2

Given GATA3 positivity, estrogen receptor and mammaglobin were negative, ruling out breast primary. Wilms tumor gene protein 1 was negative, ruling out mesothelioma. Other stains, including paired box gene 8, Sry-related HMg-Box gene 10, and thyroid transcription factor 1/Napsin A were negative, ruling out ovarian and lung primary malignancies. Further pathology consultation was sought, which reported a poorly differentiated neoplasm with abundant lymphocytic and neutrophilic infiltration along with loss of PMS2 expression with intact MSH6 expression (Figure 5).

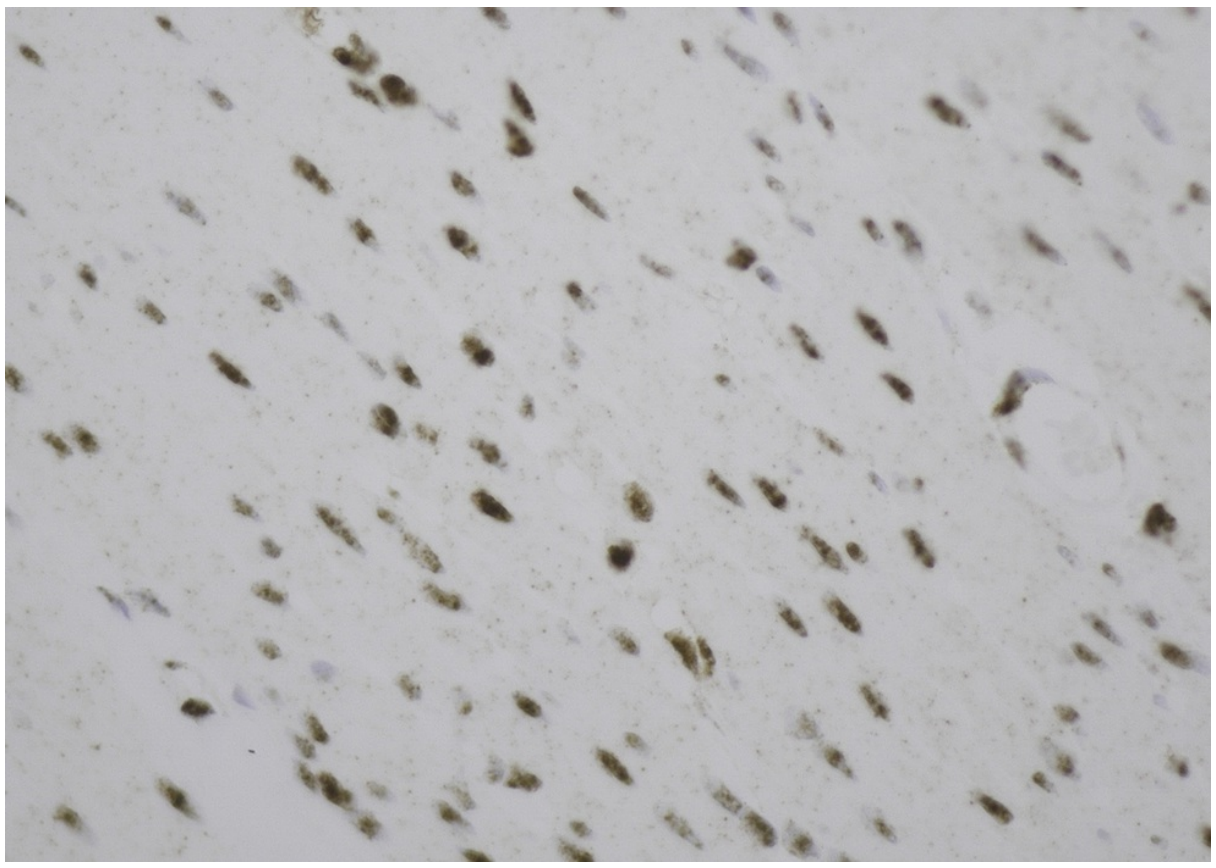

FIGURE 5: Immunohistochemistry showing loss of PMS2. 


\section{Discussion}

$\mathrm{MC}$ of the colon is a rare histologic subtype of colorectal malignancy. Only 5-8/10,000 colon cancers have the characteristic histological features of MC [4]. It is slightly more common among middle-aged women and usually presents in the proximal colon than the distal, with rare metastasis to lymph nodes. In a prospective study of 11 patients with MC, most patients had signs of a lower gastrointestinal malignancy, such as melena, abdominal pain, diarrhea, or altered bowel habits, with only one patient developing an abdominal mass [5]. Our patient also presented with nonspecific symptoms, including abdominal pain and weight loss. She also had involvement of the right side of the colon, as seen in most cases [2].

MC was previously called "large cell minimally differentiated carcinoma" as it is morphologically similar to undifferentiated carcinoma. Tumor cells in MC are highly proliferative with abundant cytoplasm and prominent nucleoli and can invade surrounding structures. The World Health Organization further defines that cells in MC are arranged in sheets and lack the glandular formation of colonic adenocarcinoma $[6,7]$. Definitive diagnostic criteria are lacking, and the various histopathological and immunohistochemical diagnostic criteria vary depending on experience. In our case, histology was nonspecific, showing poorly differentiated malignant cells, thus increasing the diagnostic challenge.

Histology alone cannot always differentiate MC from poorly differentiated adenocarcinoma as both tumor cells show a lack of glandular structure. Therefore, IHC staining is crucial in confirming the diagnosis of MC. MC generally has a high level of microsatellite instability (MSI) and loss of mutl homolog1 (MLH1) [3]. It frequently does not express specific stains for intestinal differentiation such as CDX2 and CK20 but can stain positive for calretinin and CK7 [7]. In our case, the pathology showed a poorly differentiated tumor that was negative for CK20 but positive for CDX2, creating a diagnostic dilemma. Calretinin positivity and loss of PMS2 helped in narrowing our differential. According to a study by Lin et al., $89 \%$ of cases were positive for cadherin (CDH17) and SATB2, while calretinin was positive in $67 \%$ of the cases. Studies have also shown that $60 \%-80 \%$ of MC are associated with deficient mismatch repair protein and a high degree of MSI. The majority of cases in the literature showed loss of both MLH1 and PMS2 [7,8]. Our case had isolated loss of PMS2 expression. Molecular testing for MLH1 methylation was not performed. Isolated loss of PMS2 is uncommon and seen in about $4 \%$ of all CRCs. These tumors may also demonstrate more aggressive behavior than other tumors with MSI [9].

Another interesting aspect of our case was the presentation of bulky abdominal lymphadenopathy, which initially favored the diagnosis of lymphoma. Our case was metastatic at presentation, which is uncommon for MC. In a population-based analysis conducted by Thirunavukarasu et al., out of 74 patients diagnosed with MC, only $10 \%$ presented with metastatic disease [10]. Among the 11 patients studied by Jessurun et al., none were metastatic at the time of diagnosis, and only one patient had liver metastasis at the time of presentation [5]. Studies have shown that MC has a favorable prognosis than poorly differentiated and undifferentiated adenocarcinoma [11]. Although it is unclear why MC has improved overall survival, one possibility could be due to the low incidence of metastatic disease at presentation. One study found that patients with MC have five-year mortality of $40 \%$ compared to $59 \%$ in poorly differentiated adenocarcinoma [12]. According to a meta-analysis of 16 studies, MC has a lower incidence of lymph node involvement than poorly or undifferentiated adenocarcinoma [1]. MC is treated similarly to colon adenocarcinoma. Surgical resection is used to treat localized disease, while chemotherapy is used to treat advanced colon cancer. In a recent phase three study of pembrolizumab versus chemotherapy as first-line therapy for MSI-high advanced CRC, the pembrolizumab group had better progression-free survival (16.5 versus 8.2 months) and fewer treatment-related adverse events [13]. Recent literature has shown that compared with other microsatellite-stable and microsatellite-unstable CRC, tumor cells in MC have a higher number of CD8+ cytotoxic T cells and upregulation of programmed cell death protein 1 (PD-1) and interferon-gamma genes. Upregulation of PD-1 can have potential therapeutic benefits in the future with anti-PD-1 therapy [14]. Our patient, unfortunately, was not tested for PD-1. Knox et al. conducted a retrospective analysis of 102 patients with MC and found that BRAFV600E mutation was more common in MC (86\%), and these patients were at high risk for Lynch syndrome [11]. Our patient had poor performance status with the Eastern Cooperative Oncology Group of 2-3. The patient and family eventually opted for the best supportive care and hospice. Further molecular testing including next-generation sequencing was not performed. However, based on the KEYNOTE-177 trial mentioned above, the patient would have been a candidate for anti-PD-1 therapy with pembrolizumab. Further studies are needed to investigate different treatment approaches and disease response.

\section{Conclusions}

In conclusion, $\mathrm{MC}$ of the colon differs from the adenocarcinoma in terms of clinical and immunohistochemical characteristics. Due to its atypical histopathological presentation, immunohistochemical staining and morphological diagnosis are needed for accurate diagnosis. In addition, it is very commonly associated with high MSI. MC has a better prognosis than poorly differentiated adenocarcinoma. Further studies are needed to elucidate the molecular analysis and treatment regimen for MC. 


\section{Additional Information}

Disclosures

Human subjects: Consent was obtained or waived by all participants in this study. Conflicts of interest: In compliance with the ICMJE uniform disclosure form, all authors declare the following: Payment/services info: All authors have declared that no financial support was received from any organization for the submitted work. Financial relationships: All authors have declared that they have no financial relationships at present or within the previous three years with any organizations that might have an interest in the submitted work. Other relationships: All authors have declared that there are no other relationships or activities that could appear to have influenced the submitted work.

\section{References}

1. Pyo JS, Sohn JH, Kang G: Medullary carcinoma in the colorectum: a systematic review and meta-analysis . Hum Pathol. 2016, 53:91-6. 10.1016/j.humpath.2016.02.018

2. Martinotti M, Cirillo F, Ungari M, et al.: Microsatellite instability in medullary carcinoma of the colon . Rare Tumors. 2017, 9:6541. 10.4081/rt.2017.6541

3. Cunningham J, Kantekure K, Saif MW: Medullary carcinoma of the colon: a case series and review of the literature. In Vivo. 2014, 28:311-4.

4. Mitchell A, Bendavid Y: Medullary colon cancer presenting with total necrosis of all regional lymph node metastases: morphologic description of a presumed immune-mediated event. Diagn Pathol. 2014, 9:204. 10.1186/s13000-014-0204-X

5. Jessurun J, Romero-Guadarrama M, Manivel JC: Medullary adenocarcinoma of the colon: clinicopathologic study of 11 cases. Hum Pathol. 1999, 30:843-8. 10.1016/s0046-8177(99)90146-6

6. Winn B, Tavares R, Fanion J, Noble L, Gao J, Sabo E, Resnick MB: Differentiating the undifferentiated: immunohistochemical profile of medullary carcinoma of the colon with an emphasis on intestinal differentiation. Hum Pathol. 2009, 40:398-404. 10.1016/j.humpath.2008.08.014

7. Gómez-Álvarez MA, Lino-Silva LS, Salcedo-Hernández RA, et al.: Medullary colonic carcinoma with microsatellite instability has lower survival compared with conventional colonic adenocarcinoma with microsatellite instability. Prz Gastroenterol. 2017, 12:208-14. 10.5114/pg.2016.64740

8. Lin F, Shi J, Zhu S, et al.: Cadherin-17 and SATB2 are sensitive and specific immunomarkers for medullary carcinoma of the large intestine. Arch Pathol Lab Med. 2014, 138:1015-26. 10.5858/arpa.2013-0452-OA

9. Alpert L, Pai RK, Srivastava A, et al.: Colorectal carcinomas with isolated loss of PMS2 staining by immunohistochemistry. Arch Pathol Lab Med. 2018, 142:523-8. 10.5858/arpa.2017-0156-OA

10. Thirunavukarasu P, Sathaiah M, Singla S, et al.: Medullary carcinoma of the large intestine: a population based analysis. Int J Oncol. 2010, 37:901-7. 10.3892/ijo_00000741

11. Knox RD, Luey N, Sioson L, et al.: Medullary colorectal carcinoma revisited: a clinical and pathological study of 102 cases. Ann Surg Oncol. 2015, 22:2988-96. 10.1245/s10434-014-4355-5

12. Wick MR, Vitsky JL, Ritter JH, Swanson PE, Mills SE: Sporadic medullary carcinoma of the colon: a clinicopathologic comparison with nonhereditary poorly differentiated enteric-type adenocarcinoma and neuroendocrine colorectal carcinoma. Am J Clin Pathol. 2005, 123:56-65.

13. Shiu KK, Andre T, Kim TW, et al.: KEYNOTE-177: phase III randomized study of pembrolizumab versus chemotherapy for microsatellite instability-high advanced colorectal cancer. J Clin Oncol. 2021, 39:6-6. 10.1200/JCO.2021.39.3_suppl.6

14. Friedman K, Brodsky AS, Lu S, et al.: Medullary carcinoma of the colon: a distinct morphology reveals a distinctive immunoregulatory microenvironment. Mod Pathol. 2016, 29:528-41. 10.1038/modpathol.2016.54 